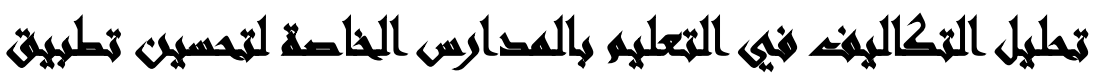

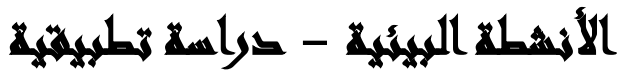

\section{[10]}

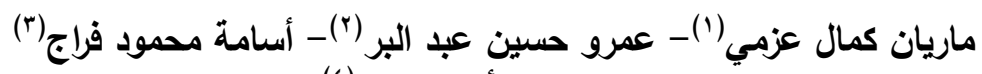

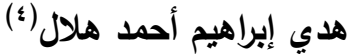

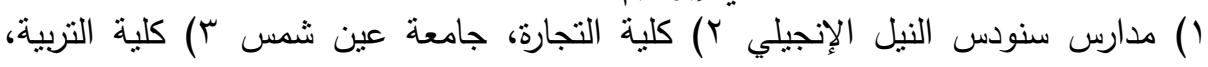
جامعة القاهرة ع) معهد الدراسات والبحوث البيئية، جامعة عين شمس الئه

\section{المستخليس}

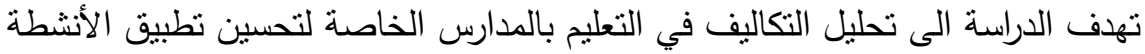

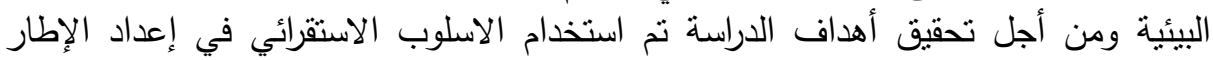

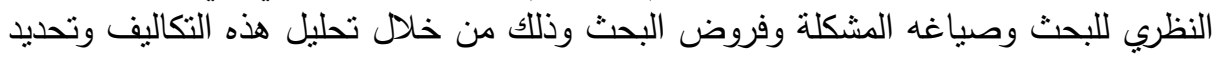

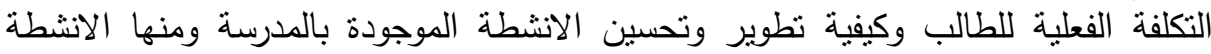

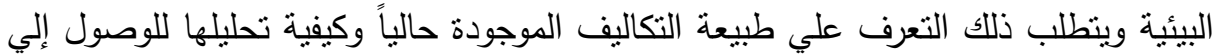
تكلفة الطالب الفعلية. وتم استخدام التحليل الاحصائي وذللك للتأكد من صحة التهة الفروض الدراسة.

وقد توصلت الدراسة الى النتائج الآتية: وجود علاقة ذات دلالة احصائية بين تكلفة

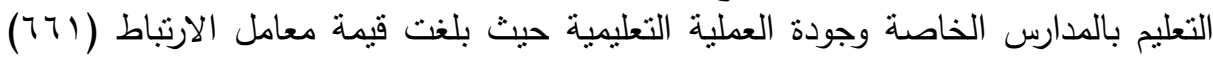
عند مسنوى دلالة ا..,... ووجود علاقة ارتباط ذات دلاله إحصائية بين تكلفة الطالب الفية الفعلية

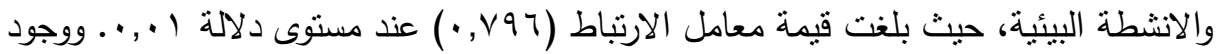

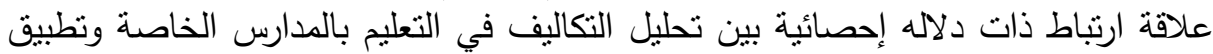

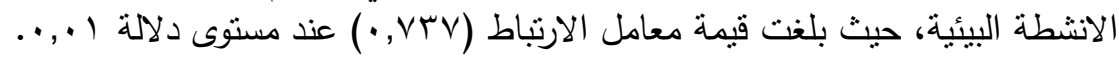

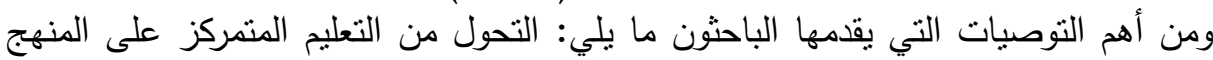

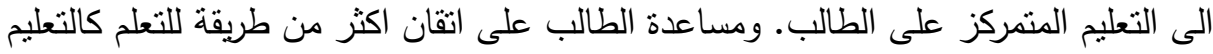

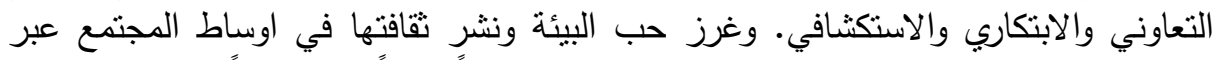

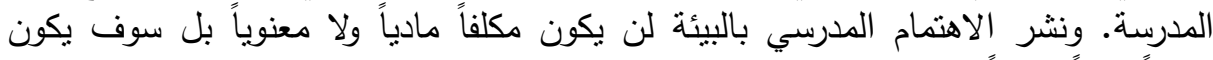

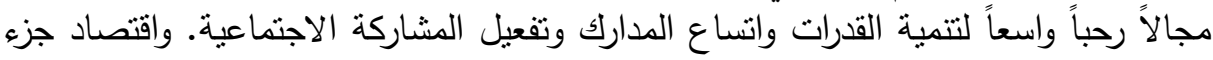
من تكاليف الانشطة التعليمية والانشطة المدرسية لأنشطة البيئة المتعددة.

$$
\text { المجلد الخامس والأربعون، الجزء الثانى، مارس } 19
$$




\section{הهبه}

يعد التعليم هو النشاط الذي يهدف إلى المعرفة والقيم الروحية والفهم والإدراك الذي

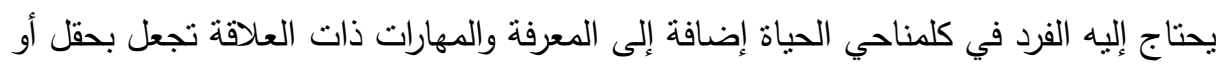
مجال محدد للتعليم أهمية كبيرة في المجتمع إذ يعطي للثخص المتعلم الاحترام، قيمة رفيعة، إنهاء

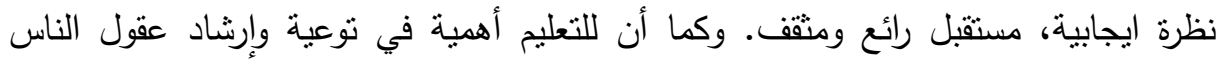
ويهدف إلى التتمية العقلية للارسين بصورة واسعة، ومن شأنه تقوية مهارات الإنسان الذي ولئي يحتاجها للاستمرار في حياته وتخطي جميع المراحل. إن العلم هو نور للإنسان ويبعث

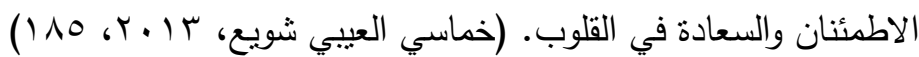
ويتبين من خلال دراسة الوضع الراهن للتعليم الخاص في مصر أن عدد المدارس

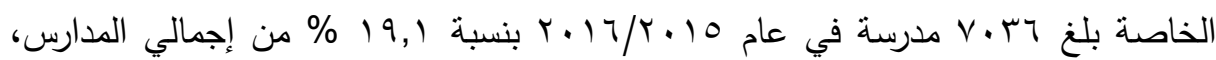

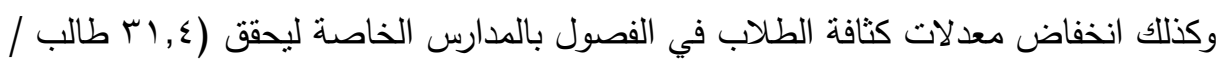
فصل) ليقترب بذلك من الحد الأقصى للمعدل العالمي (•r طالب / فصل). كما أوضحت الدراسة أن المدارس الخاصة يتركز نشاطها بشكل كبير في مراحل التعليم ما قبل الابتدائي

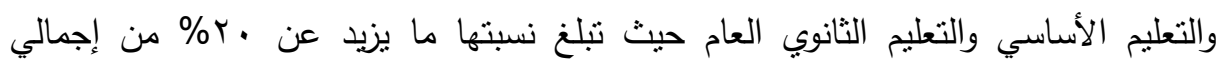

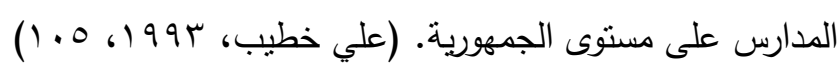

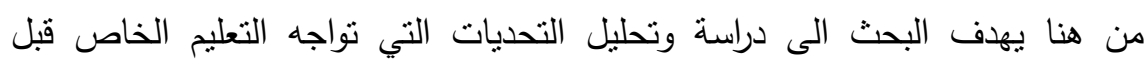
الجامعي هي اهتمام أصحاب المدارس الخاصة بشكل أكبر بمعايير الربحية مقارنة بمعايير جودة العملية التعليمية، بالإضافة إلى حاجة التعليم الخاص إلى استثمارات كبيرة لإنثاء المدارس واحتدام حدة المنافسة بين المدارس الخاصة العادية والدولية على جذب الطلاب.

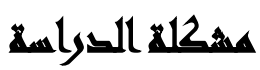

تتمثل مشكلة البحث في وجود تكاليف في التعليم بالمدارس الخاصة والرغبة في تحسين تطبيق الانشطة البيئية وذلك عن طريق تحليل هذه التكاليف وتحديد التكلفة الفعلية للطالب بلبئ وكيفية تطوير وتحسين الانشطة الموجودة بالمدرسة ومنها الانثطة البيئية (عبد الله غالب عبد بلئ

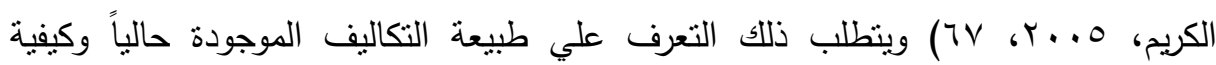
416

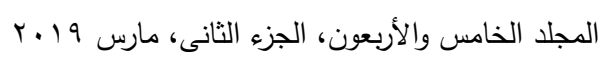


تحليلها للوصول إلي تكلفة الطالب الفعلية حتي يتم الحصول على ميزة تتافسية بين المدارس

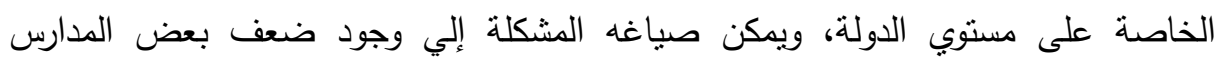

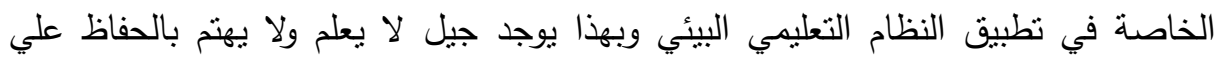

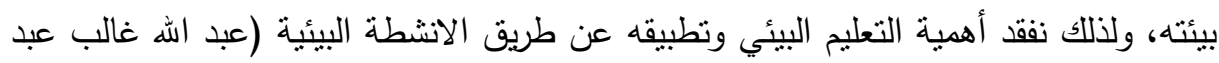

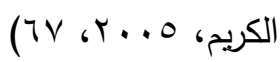

ومن خلال اطلاع الباحثون على الدراسات السابقة، مثل دراسة Abdul Ghafoor اهتمت التحقيق في سبب تراجع نظام المدارس الحكومية، مقارنة جودة التعليم

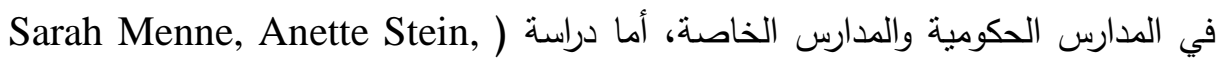
2012) فقد اهتمت بالتعرف على الصلة بين التعليم والفوائد الفردية والمجتمعية. أما دراسة وراسة (Brian A. Jacob and Jens Ludwi, 2002) تؤدى إلى كيفية تحسين نتائج التعليم للفقراء. ولكن لم يتطرق أحد الباحثون لدراسة التحديات

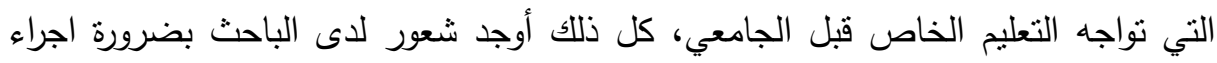
دراسة لتحليل تلك التحديات ودراستها واقتراح طرق لمواجهتها أما دراسة تكلفة وعائد التعليم والفرصة البديلة (ناهد علي فاروق) جامعة السودان للعلوم والتكنولوجيا -كلية الدراسات التجارية 2015 (Vol. 16) Journal of Economic Sciences الدارس للمفاضلة بين مواصلة التعليم وعائد الفرصلة البديلة. وجد أن اجر الخريج لا يتتاسب مع نفقات الاستثمار في التعليم. أما دراسة تكاليف وتحليل وتمويل التربية وتحديد تكلفة التلميذ الواحد (طوطاوي، مبدوعة زوليخة، كآروشة قرمية) ج 1 ـ ـ r. تحديد كلفة التلميذ نسبة إلى نفقات المستخدمين الفعلية.

\section{أسئلا التوراسة}

السؤال الرئيسي: هل يمكن تحليل التكاليف في التعليم بالمدارس الخاصة لتحسين تطبيق

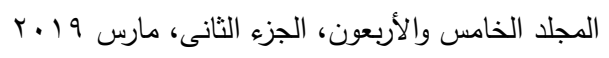




\section{يستمد من هذا السؤال عدة اسئلة أخرى منها:}

ا ـ ما طبيعة التكاليف في المدارس الخاصة وكيفية تحليلها؟

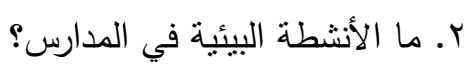

r. ما امكانية تحسين جودة الأنشطة البيئية بالمدارس؟

ـ. ما مدى وجود جدوى من تفعيل تطبيق نظام الأنشطة البيئية؟

\section{أهمية التصاسة}

يمكن تحديد الأهمية العلمية والتطبيقية على النحو التالي:

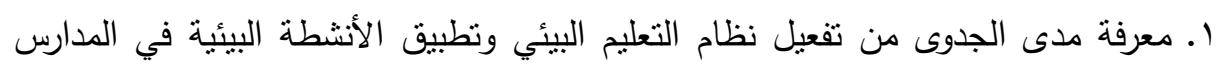

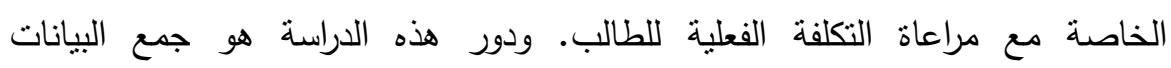
والمعلومات اللازمة عن هذه الدراسة المتاحة.

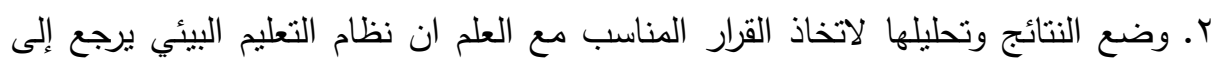
الفهم وتعلم الوعي والمعرفة البيئية الأساسية بهدف تتمية السلوك البيئي الايجابي والدائم

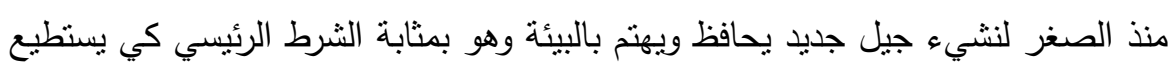

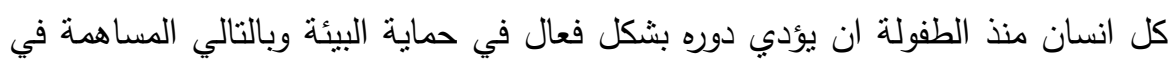
الحفاظ على صحته من خلال محافظته وبيئته المحلية وهذا يؤدي الي حفاظه على البيئة المصرية كلها.

r. وهنا نرى أهمية التعليم البيئي في المدارس الخاصة للأطفال وأيضاً دور التدريب للقائمين على العملية التعليمية في هذا المجال فعند دخول هذا التعليم في المدارس تتحول إلى ثقافة فئن

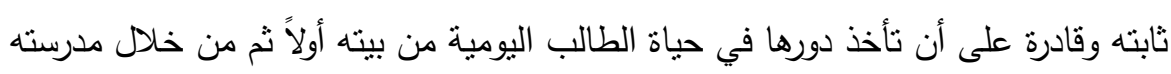

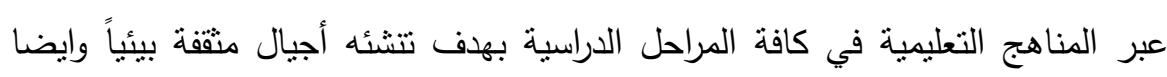
يؤثز هذا التعليم على كل العاملين بالمؤسسات التعليمية فنؤثر بشكل جيد وفعال في كافة فئة فئه النشاطات البشرية. وفي ضوء أهمية الدراسة يتم تحديد التكاليف في التعليم بالمدارس باس فئنس الخاصة لتطوير وتحسين الانشطة البيئية في كل من (التدريب - الابتكار - حماية البيئة

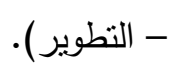


مجلة العلوم البيئية

معهد الدراسات والبحوث البيئية - جامعة عين شمس لهن لهن

\section{أهساهنم السراسلة}

الهذف الأساسي للاراسة هو: تحليل التكاليف في التعليم بالمدارس الخاصة لتحسين تطبيق

الأنشطة البيئية. ويتحقق من خلال الأهداف الفرعية التالية:

( ) تحديد وتحليل التكاليف في المدارس الخاصة.

r) تطوير الأنشطة البيئية بالمدارس.

؟) دور التدريب في تتمية العملية التعليمية.

؟) دور المقررات التعليمية في تحسين جودة الأنشطة البيئية.

\section{هزوضر القواسمة}

تم صباغة الفروض التالية كأسباب محتملة للمشكلة محل الدراسة كالتالي: الفرض الرئيسي الأول: تم رفض الفرض القائل بأنه "لا توجد علاقة جوهرية ذات دلاله

إحصائية بين تكلفة المدارس الخاصة وجودة العطلية التعليمية". الفرض الرئيسي الثاني: نم رفض الفرض القائل بأنه "لا توجد علاقة جوهرية ذات دلاله

إحصائية بين تكلفة الطالب الفعلية وتكلفة الانشطة البيئية". الفرض الرئيسي الثالث: تم رفض الفرض القائل "لا توجد علاقة ذات دلاله إحصائية بين تحليل التكاليف في التعليم بالمدارس الخاصة وتطبيق الانشطة البيئية".

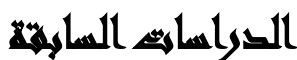

أولاً: الدراسات السابقة العريبة: دراسة (منى عمر محمد مصطقى، ب ( ب r): وقد هدفت الدراسة الى التعرف على دور الانفاق العام على التعليم في رفع جودته في مصر • وبيان مدى إمكانية استخدام نظام تكاليف

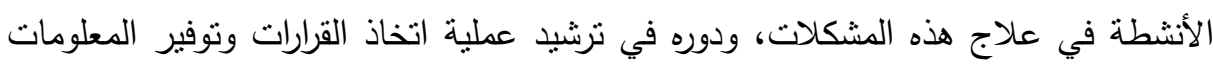
اللازمة، لتسعير تلك الخدمات.

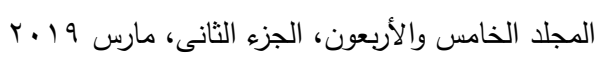


دراسة (عبير عبد المنعم، 10 ب): وقد هدفت الدراسة الى التعرف على دور الادارات التعليمية في تطوير بيئة المدرسة وتتمية السلوك البيئي لدى التلاميذ مرحلة التعليم الاساسي.

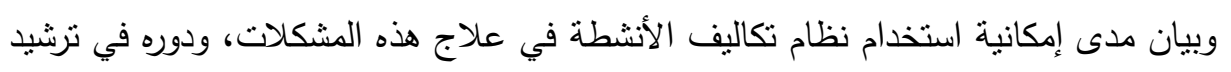

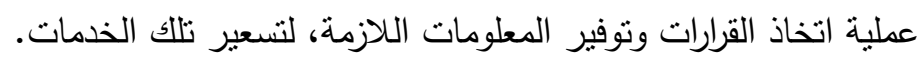
دراسة (سالم عبد الله حس، 17 ب ب): وقد هدفت الدراسة الى التعرف على دراسة وتحليل نظام تكاليف الأنشطة من حيث مفهومه ومقوماته وخصائصه واستخداماته.

دراسة (ناهد علي فاروق): عن أهم مشكلات قياس وترشيد التكاليف في مجال الخدمات

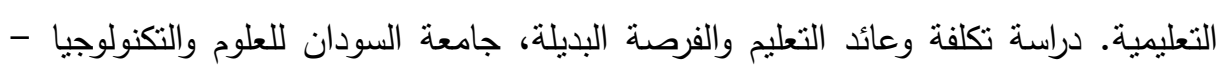
كلية الدراسات التجارية 2015 Journal of Economic Sciences (Vol. 16) بناء نموذج رياضي يعتمدها لدارس للمفاضلة بين مواصلة التعليم وعائد الفرصة البديلة. وجد أن

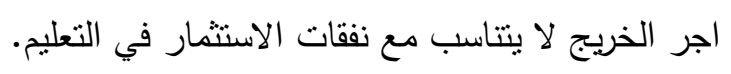

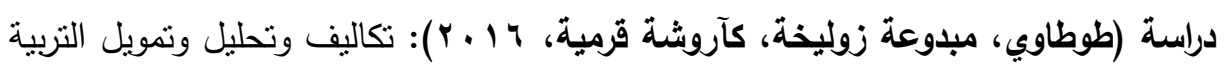
وتحديد تكلفة التلميذ الواحد - تحديد كلفة التلميذ نسبة إلى نفقات المستخدمين الفعلية. ثانياً: الاراسات السابقة الأجنبية: دراسة (Abdul Ghafoor Awan, 2015): وقد هدفت الدراسة الى: • التحقيق في سبب تراجع نظام المدارس الحكومية. • مقارنة جودة التعليم في المدارس الحكومية والخاصة.

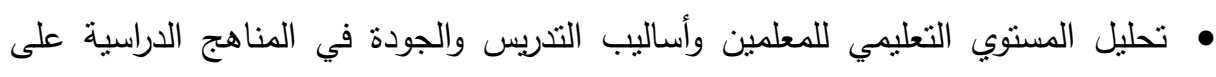
مسنوي المدارس الخاصة والمدارس الحكومية. • تحليل التحديات المستقبلية لكل من أنظمة المدارس الحكومية والمدارس الخاصة.

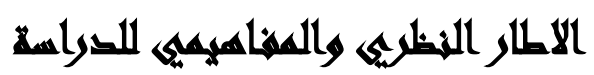

تتاولت الدراسة مجموعة من المفاهيم بالتوضيح أهمها ( المدارس الخاصة، التكاليف في المدارس الخاصة، تكلفة التعليه، البيئة، التربية البيئية، النشاط البيئي). 


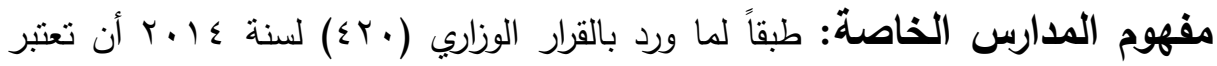

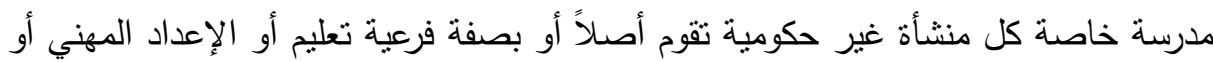
John B. ) الفني قبل مرحلة التعليم الجامعي مقابل مصروفات تعتبر مدرسة خاصة.

(Horowitz, 2004, 56 مفهوم التكاليف في المدارس الخاصة: هي كافة النفات التي تتفقها الددرسة في استمرار أداء رسالتها، يقوم نظام التكاليف في المدارس الخاصة علي تبويب عناصر التكاليف التي تتفق في المدارس الخاصة إلي عناصر معينة نسهل استخراجها. (عبير عبد المنعم،

\section{مفهوم تكلفة التعليم في المدارس الخاصة:}

- مقياس لمقار الإنفاق النقدي وغير النقدي الذي يتم في سبيل تحقيق منفعة محددة.

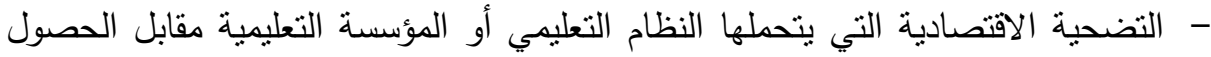
على خدمة أو منفعة ضرورية طبقاً للمعايير المقررة ويمكن قباسها في شكل وحدات نقدية. - مجموع النفقات المالية الحالية أو المتوقع إنفاقها لإنتاج مخرجات معينة من القوي البشرية المجهزة بالمعارف والمهارات والاتجاهات الأساسية التي تمكنهم من التكيف مع الحياة

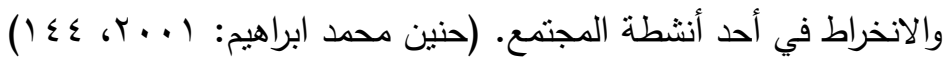

- المصادر الطبيعية لذا فأن مفهوم بيئة الإنسان محددة ولهذا فأننا لا نسنطيع تحديدها ومعالجتها كونها فكرة معينة محددة تعمل في مجال منعزل عن باقي مجالات حياة الإنسان

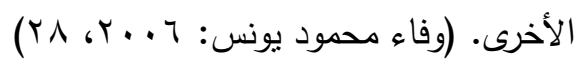

مفهوم التربية البيئية: هي جهد تعليمي موجه ومقصود نحو التعرف وتكوين المدركات بونيات لفهم العلاقات المعقدة بين الانسان وبيئته بأبعادها الاجتماعية والتقافية والبيولوجية والفيزيائية حتى يكون واعيا لمشكلاتها وقادرا على اتخاذ القرار نحو صيانتها والاسهام في حل مشكلاتها من اجل تحسين نوعيه الحياه لنفسه ولأسرته وللمجتمع ثم العالم ككل. لقد تنين بأن هناك جهود تتضافر من جميع المؤسسات لنشر الوعي البيئي لكن هذا الجهد التعليمي لا يكون

$$
\text { المجلد الخامس والأربعون، الجزء الثانى، مارس } 19 \text { ـ ب }
$$


اعتباطي إنما مقصود وموجه لفهم العلاقة بين الإنسان والبيئة زد على ذلك انها تساعد في

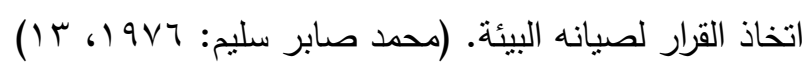
مفهوم النشاط البئي: هو مجال نعليمي صالح يمارسه الطلاب عن طريق المواقف

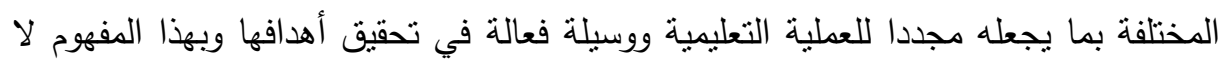
يعتبر وسيلة للعبث أو اللهو أو اللعب بل نشاطا موجها يتم بإثراف مقصود من المدرسة سواء

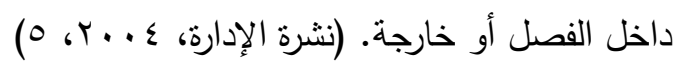

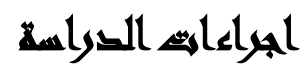

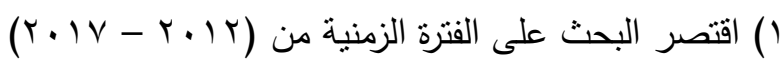

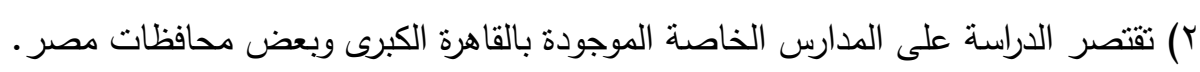

\section{منهمبية التراسة}

أسلوب الدراسة: في سبيل تحقيق هدف الدراسة واختبار فروضها اعتمد الباحثون على الأى الأسلوب الاستقرائي في جمع البيانات. ويمكن للباحث عرض منهجية الدراسة من خلال

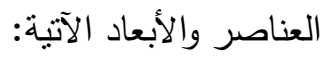

المنهج الاستقرائي: اعتمدت الدراسة علي الاسلوب الاستقرائي في إعداد الإطار النظري

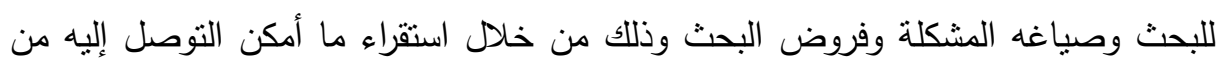
المراجع العلمية والعربية والاجنبية المتخصصة في مجال التكاليف ونظام الأنشطة البيئية. المنهج الوصفي: اعتمدت الدراسة على الاسلوب الوصفي والتحليلي واعنمدت علي المصادر الآتية: • مصادر ثانوية: استخدام المراجع والمصادر المنوفرة في المكتبات وذلك بناء علي الاطار النظري لهذه الدراسة. • مصادر أولية: دراسة تطبيقية على المدارس الخاصة. 


\section{نمأئ التصراسة}

أولاً: نتائج الدراسة التطبيقية:

( ) أصبحت المدرسة هي المؤسسة الاجتماعية التي أعدها المجتمع لتزويد الطلاب بالخبرات

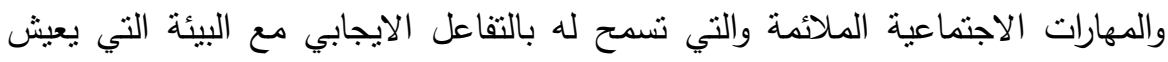
فيها.

r) الأنشطة البيئية لها دور كبير وهام في دعم العملية التعليمية وتؤثر في شخصية الطالب تأثنرا إيجابيا في قدرته على التحصيل الدراسي حيث تعمل على اتساع نقاع نقافة الطلاب

$$
\text { ومعلوماتهم وتجعله قادرا على نتظيم الوقت. }
$$

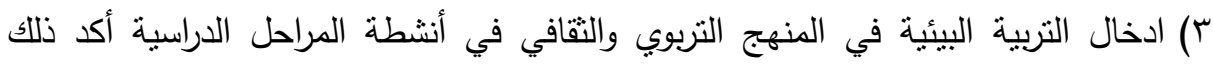
بهدف تعميق الوعي البيئي لدى التلاميذ ضرورة ملحة بحيث نسنطيع نلاك المؤسسات التربوية ترجمة الأفكار والمفاهيم البيئية إلى أنماط سلوكية تحافظ على البيئة إضافة إلى لى لئي

$$
\text { صحة وحياة الإنسان. }
$$

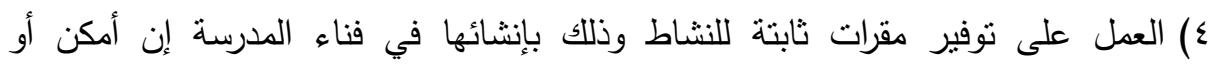
استغلال أسطح المدارس وعمل هناجر كورش للعمل ومقرات يمارس فيها النشاط وليس فئس فئنس

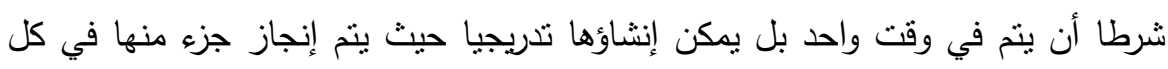

$$
\text { سنة وذللك من خلال ميزانية النشاط. }
$$

0) هناك جملة من العوامل والأسباب التي تؤدي إلى ارتفاع تكلفة التعليم لعل أهمها زيادة

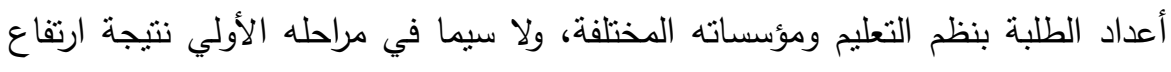

$$
\text { معدلات النمو السكاني. }
$$

؟) تزايد التوجه نحو الاستخدام المكثف لتقنيات التعليم والتعلم، ومصادر التعليم الحديثة مقابل سرعة تقادمها، وبالتالي ضرورة متابعة الجديد والمستحدث فيها من جهة وزيادة أسعارها عالمياً من جهة ثانية، مع تزايد اعتماد المؤسسات التعليمية عليها كضرورة للعملية

التعليمية التزبوية.

(اقتصاد جزء من تكاليف الانشطة التعليمية والانشطة المدرسية لأنشطة البيئة المتعددة.

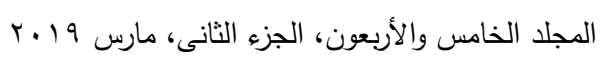




\section{ثانياً: النتائج الخاصة باختبارات الفروض:}

1. تحليل الارتباط الخطي البسيط Correlation analysis باستخدام معامل ارتباط بيرسون.

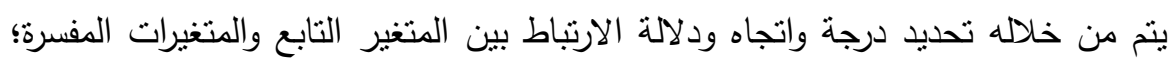
حيث يتصف معامل ارتباط بيرسون بالخصائص التالية:

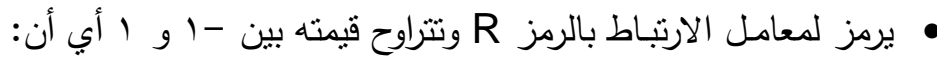

معامل بيرسون • تدل إثارة معامل الارتباط على اتجاه العلاقة، فإذا كانت الإثارة موجبة دل ذلك على إنى وجود علاقة طردية، وإذا كانت الإثارة سالبة دل ذلك على أن العلاقة العاه عكسية. • يمكن تحديد قوة العلاقة حسب القيمة المطلقة كمعامل الارتباط؛ حيث كلما اقتربت القيمة من الواحد الصحيح دل ذلك على قوة العلاقة، وكلما اقتربت من الصفر دل ذللك على الإنى

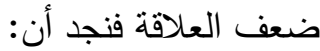

\begin{tabular}{|c|c|}
\hline القيمة & العلاقة \\
\hline 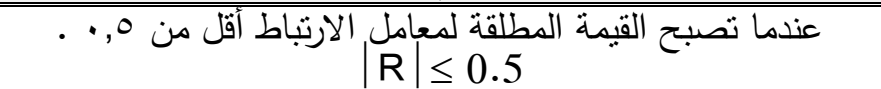 & العلاقة الضعيفة \\
\hline 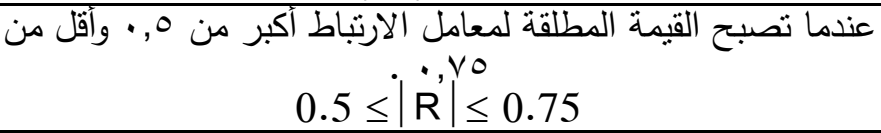 & العلاقة المتوسطة \\
\hline 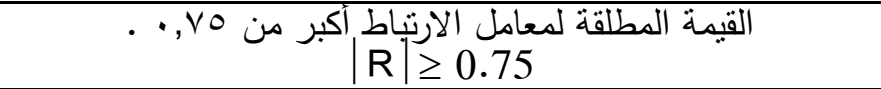 & العلاقة القوية \\
\hline
\end{tabular}

r. تحليل الانحدار المتعدد بطريقة الانحدار التدريجي واستخدام طريقة المريعات الصغرى: الانحدار التدريجي: Stepwise Regression يستخدم لتحديد أهم المتغيرات المستقلة ذات التأثير المعنوي في المتغير التابع، من خلال إدخال المتغيرات واحد بعد الأخر بخطوات متسلسلة إلى النموذج مع استبعاد المتغيرات التي تصبح غير مؤثرة بوجود بقية التئ المتغيرات، وتعتمد هذه الطريقة على إدخال جميع المنغيرات المسنقلة في النموذج كخطوة

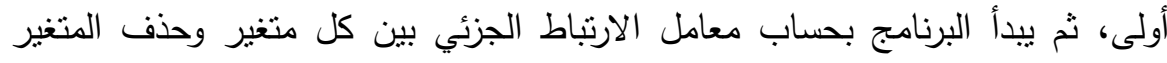

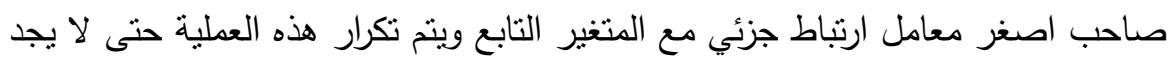
البرنامج متغير مستقل يستوفي شرط الحذف من النموذج. 
r. طريقة المربعات الصغرى تعتبر من أكثر الطرق التي استخدمت في التقدير، وهي تتطلب

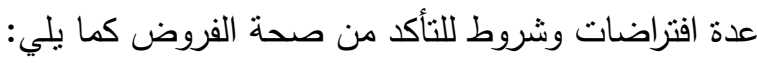
$\mathrm{Y}=\beta_{0}+\beta_{1} \mathrm{X}_{1}+\beta_{2} \mathrm{X}_{2}+\mathrm{u}$

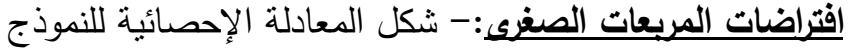

\begin{tabular}{|c|c|}
\hline المتغير التابع & $\overline{Y Y}$ \\
\hline المتغير المسنقل & $\mathrm{X}$ \\
\hline الخطاً العشوائي الغير جدير بالملاحظة. & $\mathrm{U}$ \\
\hline المقدرات & $\mathrm{B}$ \\
\hline
\end{tabular}

- العلاقة ذات اتجاه وحيد للسبيية.

- المتغيرات التفسيرية تأخذ قيمًا ثابتة من عينة لأخرى ولا يوجد بينهما علاقة خطية تامة.

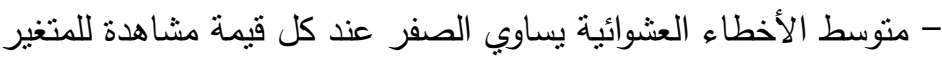
$\mathrm{E}_{\mathrm{Ut}}=0 \mathrm{t}=1 ، 2$ ، $\ldots . . . \mathrm{T}$

- نباين الأخطاء ثابت ومنساوي عند كل قيمة من قيم المشاهدة للمتغير المستقل $\mathrm{E}_{\mathrm{U}}^{2}{ }_{\mathrm{t}}=\sigma_{\mathrm{u}}^{2} t=1$ ، 2 ، $\ldots . . . T$

- عدم وجود ارتباط بين القيم المتتابعة للخطأ العشوائي عند قيم المتغير المستقل وذلك التغاير

$$
\mathrm{E}_{\mathrm{UtUs}}=0 \mathrm{t} \neq \mathrm{st} \cdot \mathrm{s}=1 \text { ، } 2 \text { ،.....T }
$$

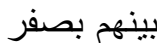

- المتغيرات التفسيرية مستقلة عن الأخطاء وذلك لأن التغاير بينهما بساوي الصفر ،

$\mathrm{E} \chi_{\mathrm{itUst}}=\chi_{\mathrm{i}} \mathrm{E}_{\mathrm{Ust}=0} \mathrm{t} \neq \mathrm{st}=1$ ، 2 ، $\ldots \ldots . \mathrm{T}$

- تؤول المتغيرات المستقلة إلى نهاية محددة المقدار عندما تؤول (t) إلى مالا نهاية

$$
\begin{aligned}
& \operatorname{Lim}_{t \rightarrow \alpha}\left(\frac{1}{T}\right) \sum_{t}^{T} X_{i t}^{2}=P \\
& \text { - عدم وجود أخطاء القياس في المنغير العشوائي. } \\
& \text { - درجات الحرية ذات قيمة موجبة (DF > O). }
\end{aligned}
$$

- استخدام أساليب التقدير العلمية بما يتفق مع النظرية الاقتصادية و المعايير الإحصائية. - تقدير تباين الخطأ العشوائي والتباين للتغيرات لتغيرات المعلمات المقدرة. 
• شـروط المربعـات الصـرى: تعد الثـروط بمثابـة إنثات لصحة فروض طريقة المربعات

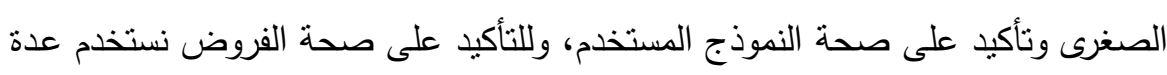

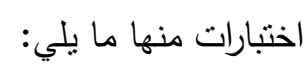

اختبر (VIF): يعد اختبار لتوضيح الازدواج الخطي بين المتغيرات التفسيرية (المستقلة)

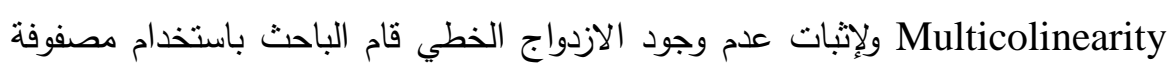

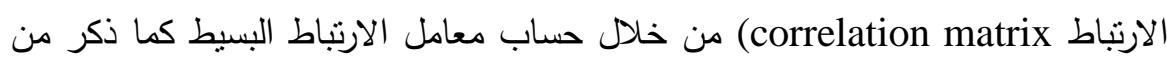
قبل ومعامل تضخم التباين (VIF)، حيث ينم حسابه لكل منغير من خلادل المعادلة

$$
V I F=\frac{1}{1-R_{i}^{2}}
$$

معامل التحديد للمتغير المستقل أ ونحصل عليه من خلال إيجاد علاقة انحدار

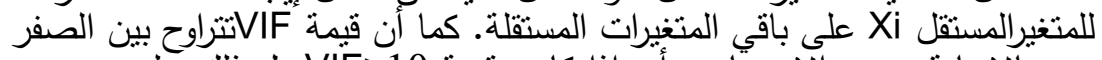

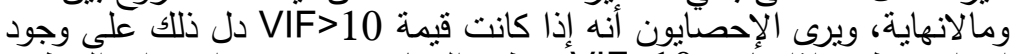

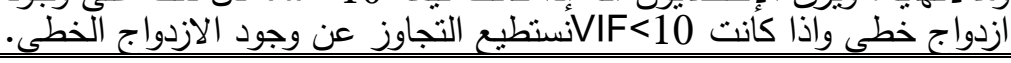

حيث:

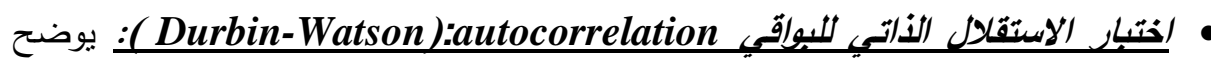
علاقة الارتباط الذاتي بين حدود الأخطاء، ويستخدم المعادلة التالية:

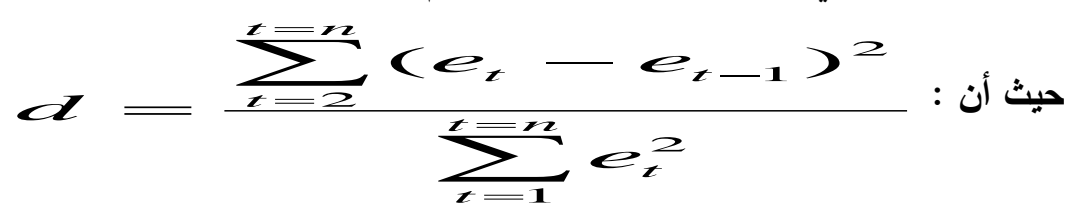

. - $e_{t-1}$ -

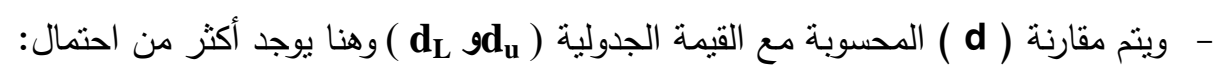
- إذا كانت إن

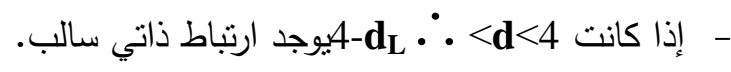
-

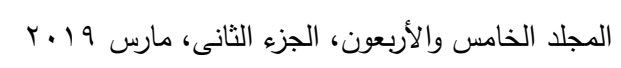


• اختبار ت (T-Test): يستخدم لحساب معنوية المتغيرات المستقلة باستخدام المعادلة

الآتية:

$$
t=\frac{\left(B_{i}\right)}{S E_{i}}
$$

حيث:

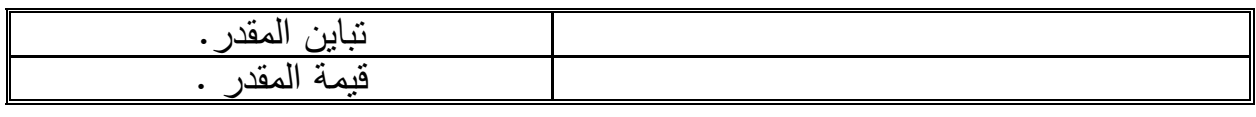

- اختبار f يستخدم لمعرفة معنوية النموذج من خلال المعادلة الآتية:

$$
\mathrm{F}=\frac{\mathrm{MST}}{\mathrm{MSE}}
$$

حيث:

MST

$$
\text { المجموعات). }
$$

دSE منوسط مجموع مربعات انحرافات منوسط كل مجموعة عن المتوسط العام (التباين

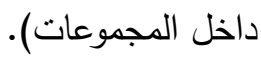

سيتم استخدام برنامج Spss Version 20 في تحليل الانحدار المتعدد بإدخال جميع

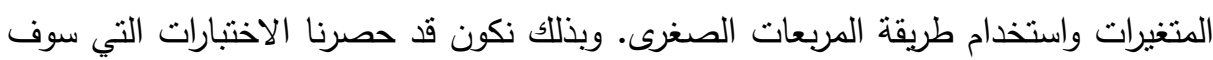
نطبقها في المرحلة القادمة على متغيرات الدراسة.

\section{الحتهار هروضر الصواسما}

اختبار صحة فرض الدراسة الأول: "لا نوجد علاقة ذات دلاله إحصائية بين تكلفة

التعليم بالمدارس الخاصةوجودة العطلية التعليمية".

وللتحقق من صحة الفرض نم استخدام معامل الارتباط البسيط Simple Correlation

coefficient، بين تكلفة التعليم بالمدارس الخاصة وجودة العملية التعليمية، والذي من خلاله يتم تحديد وجود علاقة من عدمها واتجاهها ودلالة الارتباط والجدول التالي يوضح ذلك:

$$
\text { المجلد الخامس والأربعون، الجزء الثانى، مارس } 19 \text { ـ ب ا }
$$


جدول (1): قيمة معامل الارتباط ومستوى الدلالة لتوضيح العلاقة بين تكلفة التعليم بالمدارس

\begin{tabular}{|c|c|}
\hline \multicolumn{2}{|c|}{ الخاصة وجودة العملية التعليمية } \\
\hline مستوى الدلالة & قيمة معامل الارتباط \\
\hline$\cdot, \cdot, 1$ & $\cdot, 771$ \\
\hline
\end{tabular}

يتضح من بيانات الجدول السابق وجود علاقة ذات دلالة احصائية بين تكلفة التعليم

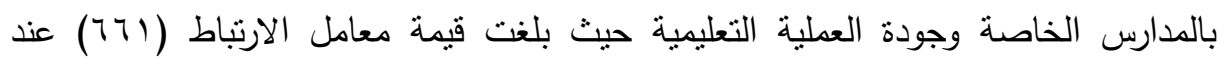

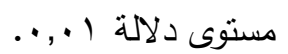

ومما سبق اتضح عدم صحة فرض الدراسة القائل "لا نوجد علاقة ذات دلاله إحصائية بين نكلفة التعليم بالمدارس الخاصة وجودة العملية التعليمية". وقبول فرض الدراسة القائل "توجد علاقة ذات دلاله إحصائية بين تكلفة التعليم بالددارس الخاصة وجودة العملية

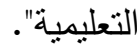
اختبار صحة الفرض الثاني: "لا نوجد علاقة ذات دلاله إحصائية بين تكلفة الطالب

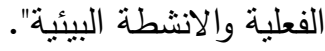

Simple Correlation وللتحقق من صحة الفرض نم استخدام معامل الارتباط البسيط coefficient، بين تكلفة الطالب الفعلية والانشطة البيئية، والذي من خلاله يتم تحديد وجود علاقة من عدمها واتجاهها ودلالة الارتباط والجدول التالي يوضح ذلك: جدول (r): قيمة معامل الارتباط ومستوى الدلالة لتوضيح العلاقة بين تكلفة الطالب الفعلية

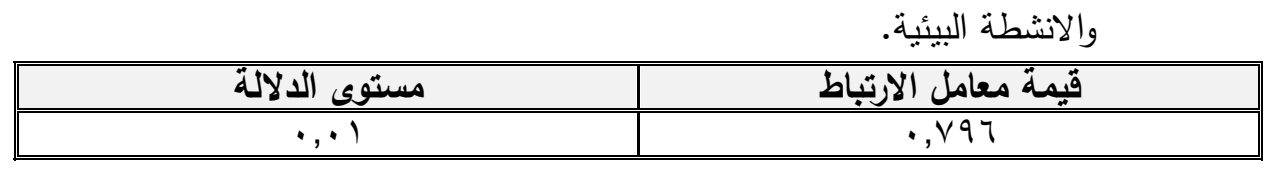
يتضح من بيانات الجدول السابق وجود علاقة ارتباط ذات دلاله إحصائية بين تكلفة

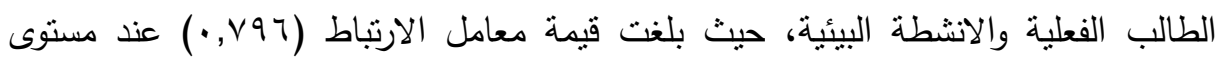

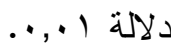

ومما سبق اتضح عدم صحة فرض الدراسة القائل "لا نوجد علاقة ذات دلاله إحصائية بين نكلفة الطالب الفعلية والانشطة البيئية وقبول الفرض البديل بوجود علاقة ذات دلاله إحصائية بين تكلفة الطالب الفعلية والانشطة البيئية".

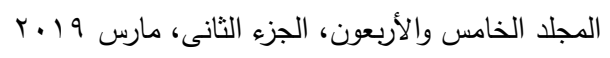


اختبار صحة الفرض الثالث: "لا توجد علاقة ذات دلاله إحصائية بين تحليل التكاليف في التعليم بالمدارس الخاصة وتطبيق الانشطة البيئية".

Simple Correlation وللتحقق من صحة الفرض تم استخدام معامل الارتباط البسيط coefficient والذي من خلاله يتم تحديد وجود علاقة من عدمها واتجاهها ودلالة الارتباط والجدول التالي

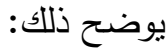
جلول (ץ): يوضح قيمة معامل الارتباط ومسنوى الدلالة لتوضيح العلاقة بين تحليل التكاليف

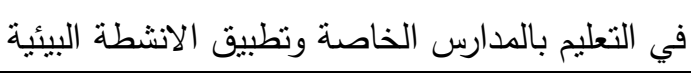

\begin{tabular}{|c|c|}
\hline مستوى الدلالة & قيمة معامل الارتباط \\
\hline$\cdot, \cdot 1$ & $\cdot, V \Psi V$ \\
\hline
\end{tabular}

يتضح من بيانات الجدول السابق وجود علاقة ارتباط ذات دلاله إحصائية بين تحليل التكاليف في التعليم بالددارس الخاصة وتطبيق الانشطة البيئية، حيث بلغت قيمة معامل

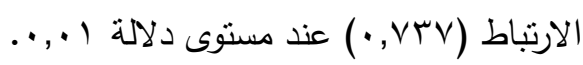

ومما سبق اتضح عدم صحة فرض الدراسة القائل "لا نوجد علاقة ذات دلالامه إحصائية

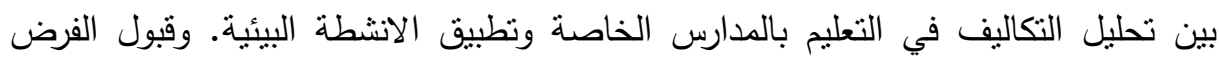

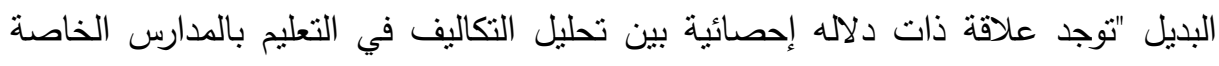
وتطبيق الانشطة البيئية". اختبار صحة الفرض الرابع: "توجد علاقة جوهرية بين تحليل التكاليف التعليم بالمدارس الخاصة وتطبيق الأنشطة البيئية وتأثنير ذلك على جودة العملية التعليمية".

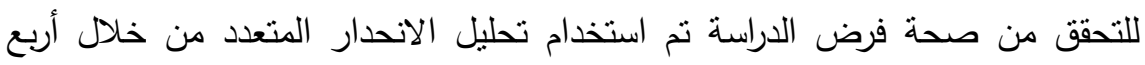

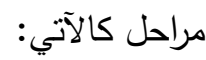
• المرحلة الأولى: صياغة النموذج. • المرحلة الثانية: تقدير المعالم.

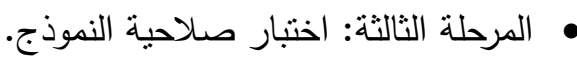


• المرحلة الرابعة: استخدام النموذج في التنبؤ •

\begin{tabular}{|c|c|c|c|c|c|}
\hline معامل التحديد & قيمة معامل & المعنوية & قيمة ت & القَّةمة & \\
\hline \multirow{5}{*}{$\cdot, 0 Y \leqslant$} & \multirow{5}{*}{$\cdot, V T V$} & $\cdot, \cdot$ & 0,191 & $V \cdot Y, 77$ & الثابت \\
\hline & & $\cdot, \cdot r$ & $r, \cdot 1$ & $\cdot, \cdots \varepsilon$ & $\mathrm{X} 1$ \\
\hline & & $\cdot, \cdot$ & r,r.r. & $\cdot, \cdot 11$ & $\mathrm{X} 2$ \\
\hline & & & & $r V, 99$ & "قيمة ف"F" \\
\hline & & & & $\cdot, \cdot$ & الدلالية \\
\hline
\end{tabular}

وسيتم استعراض تللك المراحل كما يلي:

المرحلة الأولى: صياغة النموذج: تريد الباحثة معرفة اثر العلاقة بين تحليل التكاليف التعليم بالمدارس الخاصة وتطبيق الأنشطة البيئية على جودة العملية التعليمية وبذلك يكون:

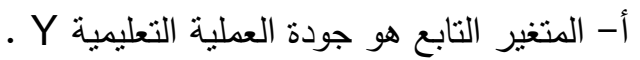
ب- المتغيرات المسنقلة تتمنل في: • المتغير المستقل الأول: تكاليف التعليم البيئية. X2. $\mathrm{Y}=\mathrm{F}\left(\mathrm{X}_{1} \cdot \mathrm{X}_{2}\right)$

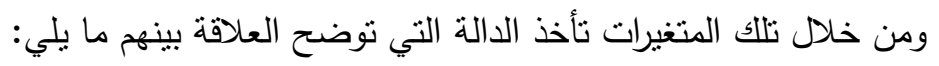

$Y=\beta_{0}+\beta_{1} X_{1}+\beta_{2} X_{2}$

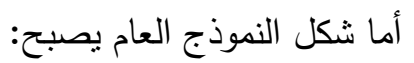

المرحلة الثانية: تقدير المعالم: جدول (ع) يوضح نتائج الانحداراتضح منبيانات الجدول السابق:

• ان قيمة معامل الارتباط الكلي بلغت VTV, • وهى دالة عند مستوى دلالة I +, • مما يدل

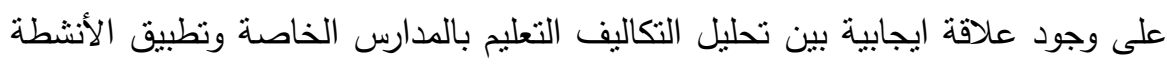
البيئية على جودة العملية التعليمية . 
• المعنوية الكلبة للنموذج: ان قيمة "ف" دالة عند مستوى دلالة ا .,.•، مما يدل على جودة

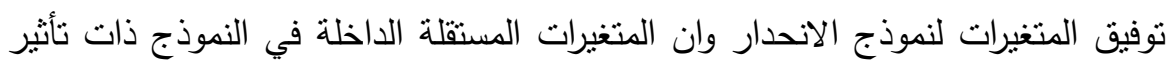
معنوي.

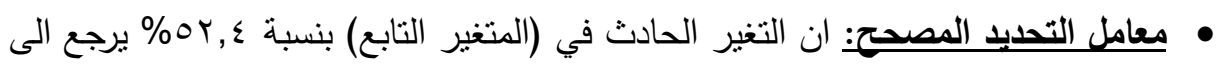
المتغيرات المستقلة الداخلة في النموذج (تكاليف التعليم البيئية والانشطة البيئية). • معنوية الجزئية (معنوية المتغيرات المستقلة): من خلال اختبار ت وهو خارج قسمة قيمة

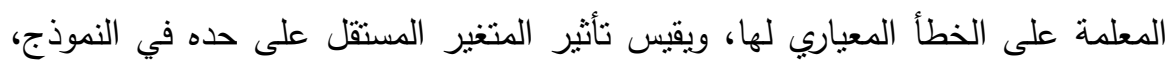

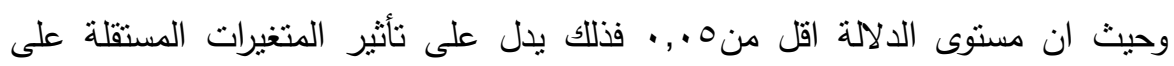
المتغير التابع

$$
Y=702.66+0.004 X_{1}+0.011 X_{2}
$$

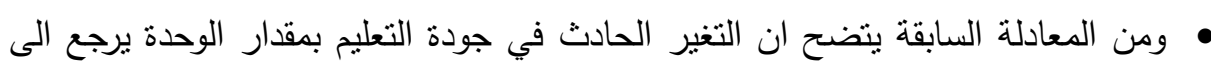

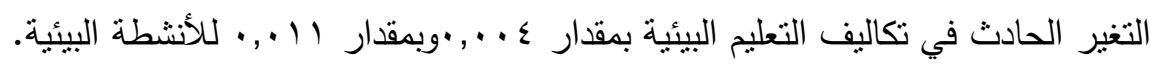

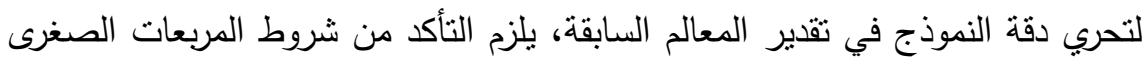

$$
\begin{aligned}
& \text { المنمثلة في الآتي: } \\
& \text { • اعتدالية التوزيع الاحتمالي للبواقي. } \\
& \text { • الاستقلال الذاتي للبواقي. } \\
& \text { • اختبار تجانس البواقي. }
\end{aligned}
$$

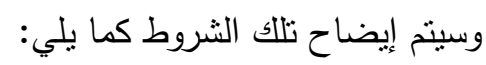

• اعتدالية التوزيع الاحتمالي للبواقي: تم التأكد لقد تم التأكد من اعتدالية التوزيع من خلال

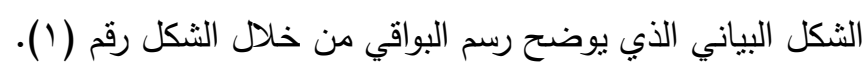

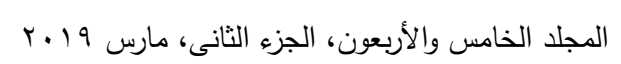




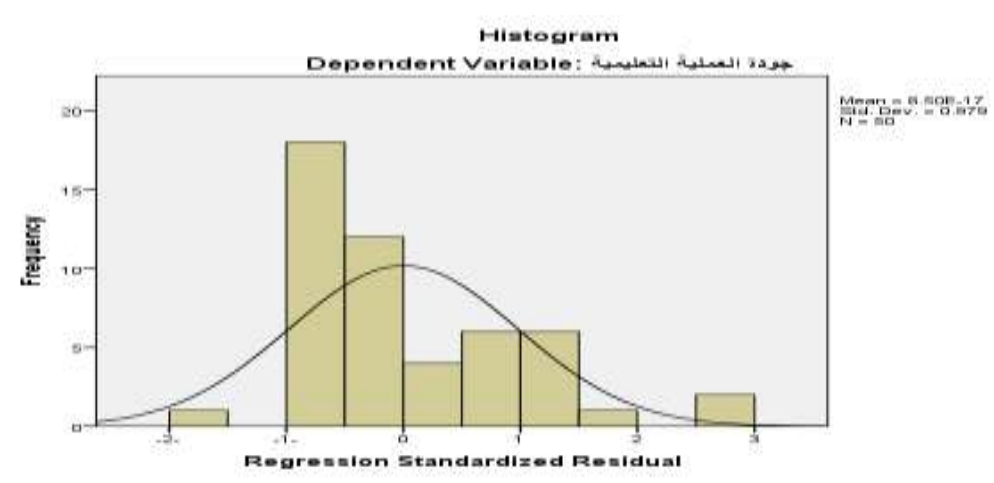

شكل (1) - (1) توزيع البواقي

من الثكل رقم (1) يتضح للباحث أن البواقي لها التوزيع الطبيعي المعياري بمنوسط

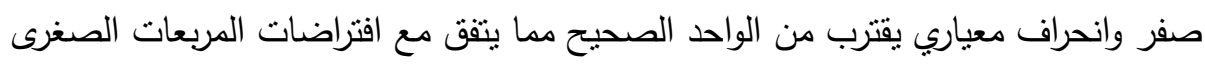

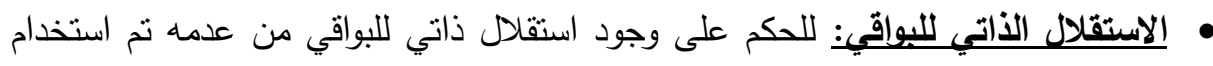
اختبار ديربن واتسون Durbin-Watson الذي يوضح علاقة الارتباط الذاتي بين حدود

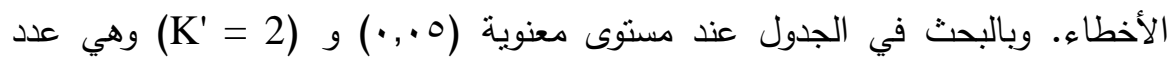

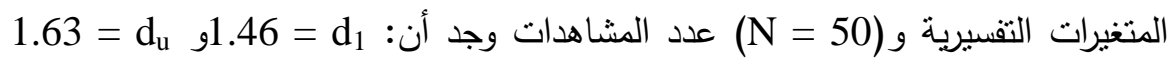
ومن نتائج النموذج اتضح أن قيمة اختبار داربن - واتسون بلغت (DW) بلغت ونظرًا لأن d المحسوبة كانت

المشكلة.

• لختبار تجانس البواقي (اختبار ثبات التبابن): استخدمت الباحثة الطريقة الاحصائية من

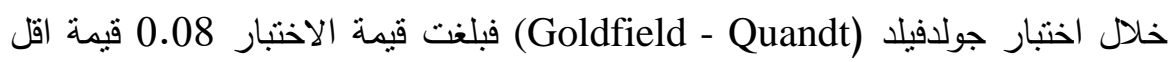

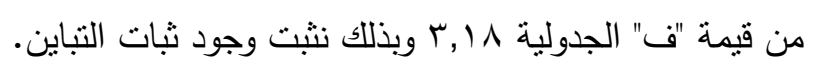
المرحلة الثالثة: اختبار صلاحية النموذج (جودة التوفيق): تم اختبار جودة التوفيق النموذج

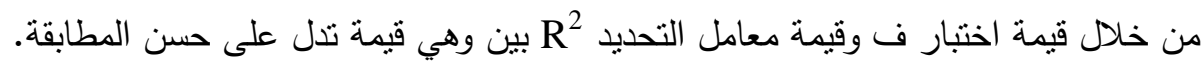

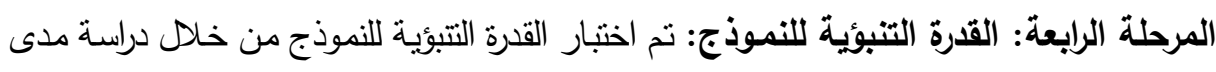

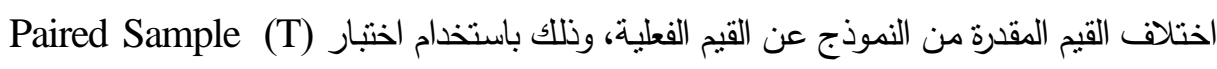
Test، فإذا جاءت فروق جوهرية دل ذلك على ضعف القدرة التتبؤية للنموذج والعكس صحيح، وعند

$$
\text { المجلد الخامس والأربعون، الجزء الثانى، مارس } 9 \text { بـ }
$$


إجراء الاختبار وجد عدم وجود فرق معنوي بينهم حيث بلغت "ت" = . ., • مما يدل على عدم وجود فرق بين القيم المقدرة والقيم الفعلية مما يؤكد قوة القدرة النتبؤية للنموذج.

\section{اللموكياهي}

من واقع النتائج السابقة ولتحقيق أهداف الدراسـة بستعرض الباحثون مجموعة من التوصيات

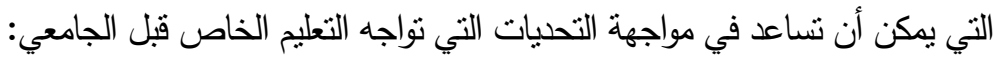
( ) التحول من التعليم المتمركز على المنهج الى التعليم المتمركز على الطالب.

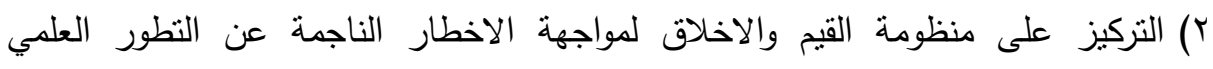
والتقني. r) مساعدة الطالب على اتقان اكثر من طريقة للتعلم كالتعليم التعاوني والابتكاري والاستكثافي. ع) ان تولي المناهج اهتماماً كبيراً بالأنشطة اللاصيفية منل زيارة النوادي العلمية والمتاحف. 0) غرز حب البيئة ونشر نقافتها في اوساط المجتمع عبر المدرسة.

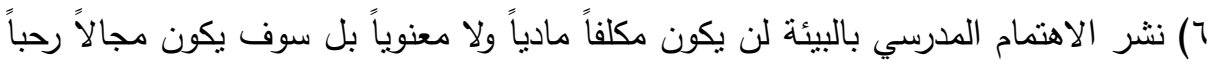
واسعاً لتتمية القدرات واتساع المدارك وتفعيل المشاركة الاجتماعية. (V اقتصاد جزء من تكاليف الانشطة التعليمية والانشطة المدرسية لأنشطة البيئة المتعددة.

\section{Inding}

استكمالاً للمسيرة العلمية اقترح الباحثون اجراء عدد من البحوث الهدف منها القاء الضوء

$$
\text { على التحديات التي تواجه التعليم الخاص قبل الجامعي ومن هذه المقترحات: }
$$

(1) يقترح الباحثون اجراء دراسة توضح العلاقة بين طبيعة المرحلة التعليمية وتكلفة التعليم.

r) اجراء دراسة توضح العلاقة بين المناهج التي تدرس وعلاقتها بكل مرحلة تعليمية. r) اجراء دراسة لمعرفة الى أي مدى تؤثر الخطة التعليمية على استيعاب الانثطة البيئية.

$$
\text { المجلد الخامس والأربعون، الجزء الثانى، مارس } 19
$$




\section{المرالئat}

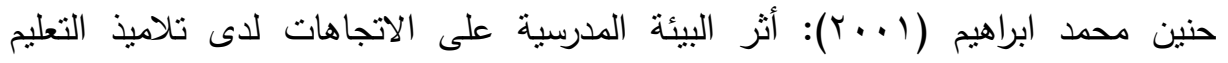

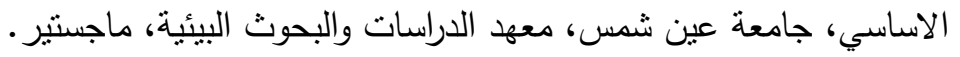

خماسي العيبي شويع: دور التعليم في تتمية الوعى البيئي (المراحل الدراسية الابتدائية

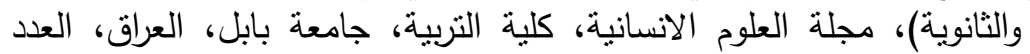

$$
\cdot(r \cdot 1 r) \text { ( } 10
$$

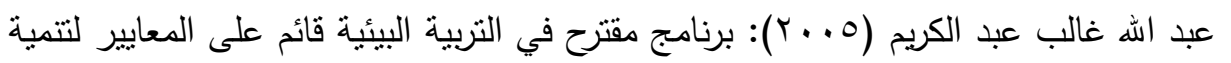

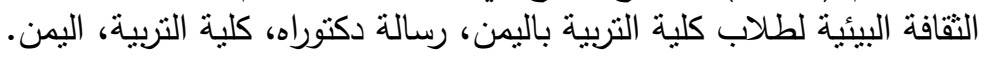

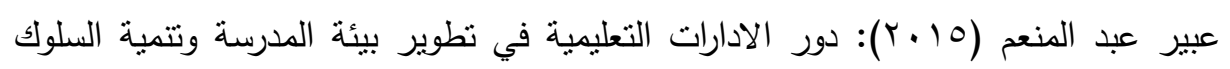

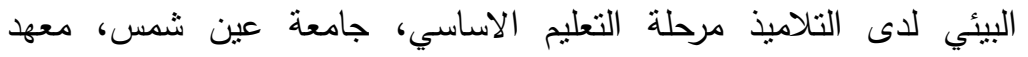

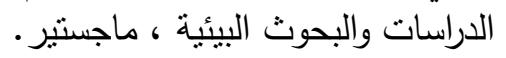

علي خطيب (ب991): التربية البيئية تعلم من اجل البيئة او تعلم للعيش في البيئة، مجلة البئة

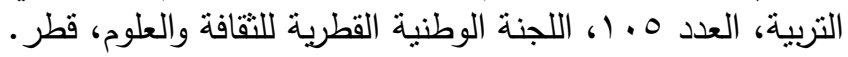

Abdul Ghafoor Awan, M. Phil (2010): Comparative Analysis of public and private Educational Institutions: A case study of District Vehari, Pakistan. Vol. 6, Issue 2, No. of periodical pages: 16.

Brian A. Jacob and Jens Ludwi (2009): National Bureau of Economic.

Sarah Menne and Annette Stein (2012): Effective Investments in Education - Background Paper, Global Economic Symposium 2012, June 11.

John B. Horowitz and Lee Specto (2016): Is there a difference between private and public education on college performance. Economics of Education, 15 July 2003; accepted 18 March 2004 Zhang Missing data imputation: focusing on single imputation. Ann Transl Med, 4(1):9.

Revista Brasileira de Pesquisa em Educação em Ciências: Environmental Education and Science Education: Ideology, Hegemony, Traditional Knowledge, and Alignment, Vol. 14, No. 2, (2014).

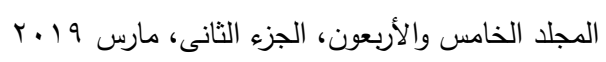


مجلة العلوم البيئية

معهد الدراسات والبحوث البيئية - جامعة عين شمس لهن لهن

\title{
COST ANALYSIS IN PRIVATE SCHOOLS EDUCATION TO IMPROVE THE APPLICATION OF ENVIRONMENTAL ACTIVITIES - APPLIED STUDY
}

\author{
Marian K. Azmy ${ }^{(1)}$; Amr H. Abdel Bar ${ }^{(2)}$; Osama M. Frag ${ }^{(3)}$ \\ and Hoda I. A. Helal ${ }^{(4)}$
}

1) Synod of Nile Evangelical Schools 2) Faculty of Commerce, Ain Shams University 3) Faculty of Education, Ain Shams University 4)Institute of Environmental Studies \& Research, Ain Shams University

\begin{abstract}
The study aims to analyze the costs of education in private schools to improve the application of environmental activities. In order to achieve the objectives of the study, the inductive method was used in preparing the theoretical framework for research, problem formulation and research hypotheses, by extrapolating as much as possible from the scientific, Environmental issues. Statistical analysis was used to ascertain the validity of the hypotheses.

The study reached the following results: there is a statistically significant relationship between the cost of education in private schools and the quality of the educational process where the correlation coefficient value (661) was at the level of significance of 0.01 . There is a significant correlation between the actual student cost and the environmental activities, where the correlation coefficient (0.796) was at the level of significance of 0.01 . There is a statistically significant correlation between the cost analysis in private schools and the application of environmental activities. The correlation coefficient (0.737) was at the mean level of 0.01 .
\end{abstract}

$$
\text { المجلد الخامس والأربعون، الجزء الثانى، مارس } 19 \text { ـ ب ا }
$$


The most important recommendations of the researcher include the following: The shift from curriculum-based to student-centered education. Focus on the system of values and ethics to face the dangers arising from scientific and technical development. Helping the student to master more than one method of learning such as collaborative, innovative and exploratory education. The curriculum should pay close attention to non-descriptive activities such as visiting scientific clubs and museums. Stimulate the love of the environment and spread its culture in the community through the school. Dissemination of school interest to the environment will not be costly materially or morally, but will be a wide area wide capacity development and widening perceptions and activating social participation. Economy is part of the costs of educational activities and school activities for multiple environmental activities. 